Morgan W.W., Keenan P.C., Kellman E.: 1943, An Atlas of Stellar Spectra, University of Chicago Press, Chicago

Oudmaijer R.D., Van der Veen W.E.C.J., Waters L.B.F.M., Trams N.R., Waelkens C., Engelsman E.: 1992, Astr. Ap. Suppl. , in press

Paresce F.: 1991, Astr. Ap. 247, L25

Renson P., Faraggiana R., Böhm C.: 1990, Bull. Inform. CDS 38, 137

Sadakane K., Nishida M.: 1986, Pub. A.S.P. 98, 685

Sargent W.L.W.: 1967 in The Magnetic and Related Stars, ed. R.C. Cameron, Mono Book Corp. Baltimore, p. 329

Stürenburg S.: 1991 in The Atmospheres of Early-type Stars, eds. U. Heber, C.S. Jeffery, Springer-Verlag Berlin, p. 229

Stürenburg S., Holweger H.: 1990, Astr. Ap. 237, 125

Trams N.R., Waters L.B.F.M., Waelkens C., Lamers H.J.G.L.M., Van der Veen W.E.C.J.: 1989, Astr. Ap. 218, L1

Venn K.A., Lambert D.L.: 1990, Ap. J. 363, 234

Waters L.B.F.M., Trams N.R., Waelkens C.: 1992, Astr. Ap., in press

\title{
DISCUSSION (Gerbaldi and Faraggiana)
}

GRIFFIN: You mentioned that one criterion of a $\lambda$ Boo star is that the Ca II K-line is very similar to that of a normal A0V star. Now, the title of this meeting is "Peculiar versus Normal Phenomena..." and the score at half-time is a complete walkover for the peculiar phenomena because the normal phenomena seem to have defaulted. Even the archetypal A0 V star Vega, which represents the basis of classification of A V stars, has been disqualified, but I'm not sure that the classification of all the other $\mathrm{A}$ dwarfs has been adjusted accordingly. The value of the K-line criterion may therefore be questionable.

GERBALDI: Yes, that is right, but the K-line criterion allows us to intercompare the classification results.

GRIFFIN: In the wide binary $r$ Per (G8 III + "A2 V"), the secondary star has been shown to have a spectrum that closely resembles that of $\lambda$ Boo. How would such a discovery affect your resolution of the question regarding the evolutionary status of the $\lambda$ Boo stars?

GERBALDI: Before drawing any conclusion we must be sure that the "A2 V" of this system is a "real $\lambda$ Boo" star, according to the criteria we gave. So, UV observations are needed.

TAKADA-HIDAI: Are there any correlations between the degree of underabundance of some species and the strength of the UV flux depressions near $1600 \AA$ and $3050 \AA$ among $\lambda$ Boo stars?

GERBALDI: The sample of $\lambda$ Boo stars analysed is very small. We have just noted the trend, that the larger the depletion in $[\mathrm{Fe} / \mathrm{H}]$, the stronger is the depression at $1600 \AA$.

SHYLAJA: The IR excess has been detected only in one case, so can it be taken as a general property of the $\lambda$ Boo stars? And have sources other than circumstellar dust been considered?

GERBALDI: The sample is much too small to be taken as representative of the $\lambda$ Boo class and to draw any general conclusion. The sensitivity of the IRAS detectors must be taken into account. The excesses observed have been interpreted in terms of black body radiation. 
CHARBONNEAU: I have a comment regarding the hypothesis of accreting depleted gas without accreting grains. This is probably possible around evolved cool stars, where grains are electrically neutral, and are rather weakly coupled dynamically to the surrounding gas. Physical conditions around an A-star are markedly different, however. In particular, there is a significant UV flux which will allow charge build-up on the grains, via photoionization. Once the grains become charged, they couple much more strongly to the gas, making it much more difficult to accrete gas without grains. I certainly agree that the accretion hypothesis is interesting and worth pursuing both from the observational and modelling standpoint. However, it is important to realize that this hypothesis has no firm physical footing as yet.

ADELMAN: Has anyone obtained two or more $I U E$ spectra of a $\lambda$ Boo star which shows the $3040 \AA$ feature?

GERBALDI: The $3040 \AA$ feature is very faint. We have not focussed our attention on it.

ARTRU: I have a comment on the identification of the $1600 \AA$ feature in $\lambda$ Boo stars. We have looked for a photoionization resonance of C I, but we did not find any at the right wavelength in the available theoretical photoionization crosssections calculated by the Opacity Project. A second difficulty for this explanation is the absence of this feature in Vega which has the same carbon abundance as $\lambda$ Boo.

BONIFACIO: On the same subject, BD $-6^{\circ} 86$ (HD 2857) is an FHB star which has been recognized to possess $\lambda$ Boo characteristics by Corbally and Gray (poster, this conference) and its UV spectrum indeed shows a shallow but very definite depression at $1600 \AA$. The interesting thing is that this star is underabundant in carbon $([\mathrm{C} / \mathrm{H}]=-2.6$; Darford and Lea, AJ 86, 1909, 1981). It seems therefore unlikely that the depression is entirely due to carbon.

LECKRONE: Snijders showed many years ago that in Vega and other stars of similar $\mathrm{T}_{\text {eff }}$ the (C I)/(C II) ionization equilibrium departs markedly from LTE. C I is over-ionized to C II, so that C I ionization edges and lines are very weak, relative to their strengths predicted in LTE. So a C I explanation for the broad $1600 \AA$ feature in $\lambda$ Boo stars is not ruled out by the absence of the feature in Vega.

COWLEY: It might be interesting for you to include the hotter, unevolved metalpoor stars in your studies. In these stars you can determine the helium abundance, and helium is very non-volatile indeed. So you might be able to establish the relation between gas-grain separation anomalies.

GERBALDI: Up to now, no $\lambda$ Boo star earlier than A0 has been detected. No $\lambda$ Boo star has been classified B9.5 by Gray, for example. 